\title{
Ultrafast dynamics of the photo-induced magneto-optical Kerr effect in CdTe at room temperature
}

\author{
A. V. Kimel, V. V. Pavlov, R. V. Pisarev, and V. N. Gridnev \\ A. F. Ioffe Physical Technical Institute, Russian Academy of Sciences, St. Petersburg 194021, Russia \\ F. Bentivegna and Th. Rasing \\ Research Institute for Materials, University of Nijmegen, Toernooiveld 1, 6525 ED Nijmegen, The Netherlands
}

(Received 12 May 2000)

\begin{abstract}
A time-resolved, all-optical technique based on the photo-induced magneto-optical Kerr effect is applied to the spectral study of the sub-picosecond, room temperature spin dynamics in CdTe between 1.44 and $1.63 \mathrm{eV}$. Two relaxation processes with respective decay times of up to $200 \mathrm{fs}$ and $2.5 \mathrm{ps}$ are distinguished. While the longer one is attributed to electron-spin relaxation, several processes such as the redistribution of electrons in the conduction band or exciton ionization near the band gap are believed to take place on the timescale of the faster one. A change of the spectral dependence of the Kerr effect from a paramagneticlike to a diamagneticlike regime during this faster relaxation is observed and discussed.
\end{abstract}

The dynamics of spins down to the subpicosecond time domain has been subject of intense research activity in recent years. ${ }^{1-6}$ In particular, the processes governing the spin coherence of excited photo-carriers and the loss of this coherence are of utmost interest, especially in semiconductors, in order to achieve ultrafast "spin electronics." Still, most of the works devoted to spin dynamics in semiconductors so far has considered low-dimensional systems at low temperatures where the behavior of the carriers is simpler to describe. However, for potential applications, room temperature studies of spin dynamics are more relevant. Beyond the gigahertz frequency domain, this dynamics can most easily be studied by optical techniques, such as polarized photoluminescence ${ }^{5}$ or magneto-optical effects. ${ }^{1,3,6}$ Both the magneto-optical Faraday effect in the transmission geometry and the magnetooptical Kerr effect in the reflection geometry are well suited to studies of the spin dynamics in a broad spectralincluding wavelengths where no luminescence can be measured-as well as thermal range. Here we report a room temperature study of the ultrafast dynamics of the photoinduced magneto-optical Kerr effect in bulk cadmium telluride (CdTe) using a sensitive, time-resolved, pump-probe technique with subpicosecond resolution. Two relaxation regimes with distinct spectral dependences were found.

Phenomenologically, optical orientation can be described as a manifestation of the inverse Faraday effect. In a static description, continuous, circularly polarized light induces a magnetization $M_{i}=\chi_{i j k}\left[D_{j} D_{k}^{*}\right]$ in a medium. ${ }^{7}$ With the use of ultrashort optical pulses, the photo-induced magnetization is transient and decays as a result of the relaxation processes. In a microscopic approach, light excites carriers with a preferential spin orientation because of the selection rules for transitions induced by circularly polarized photons. ${ }^{8,9}$ In a direct band-gap semiconductor with a zinc-blende crystallographic structure, such as CdTe, optical transitions take place around the $\Gamma$ point of the first Brillouin zone [Fig. 1(a)]. An essential feature of the band diagram is the fact that their top $p$-type valence band is split into a degenerate $P_{3 / 2}$ band-one subband for heavy-hole states, the other for light-hole states - and a split-off $P_{1 / 2}$ band that lies below $P_{3 / 2}$ at the energy $\Delta=0.9 \mathrm{eV}$ for CdTe. For photon energies equal or slightly superior to the direct band-gap energy $E_{g}$ transitions can take place between the top of the degenerate $P_{3 / 2}$ valence band at $\Gamma_{8}$ and the bottom of the $S_{1 / 2}$ conduction band at $\Gamma_{6}$. For higher photon energies, optical transitions are also possible between the top of the split-off $P_{1 / 2}$ band at $\Gamma_{7}$ and $\Gamma_{6}$ [Fig. 2(b)]. The probability of a transition involving light holes to occur is three times smaller than that of a transition involving heavy holes. For that reason, only transitions from the heavy-hole band will serve as a basis for the following discussion and figures. As the lifetime of excitons can be comparable with the pulse duration, ${ }^{10}$ transitions involving the creation of an exciton have to be taken into account.

The magneto-optical Kerr effect arises from the difference between the optical coefficients of a material for righthanded and left-handed circularly polarized light. This difference can be induced by an applied magnetic field, a spontaneous, or an optically induced magnetization. In either way, the degeneracy of the magnetic quantum number $m$ is lifted. The simulated spectral dependence of the Kerr rotation and ellipticity, together with the corresponding schematic energy level configuration, are shown in Fig. 2(b) for a two-level system. ${ }^{11}$ This type of behavior, due to the splitting (a)

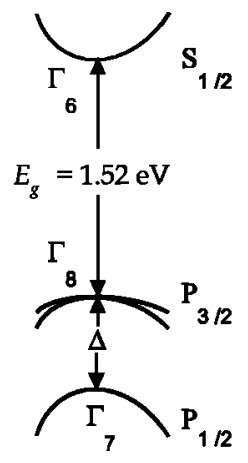

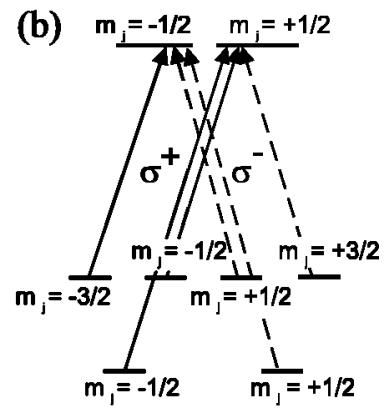

FIG. 1. (a) Schematic electronic band structure of CdTe near the direct band-gap and (b) allowed electric dipolar transitions for right-handed (solid arrows) and left-handed (dashed arrows) circularly polarized light (Ref. 8). 

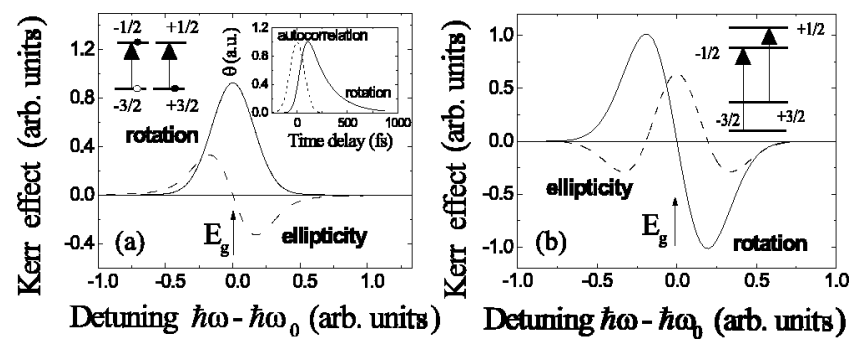

FIG. 2. Schematic optical transition configuration and simulated spectra of (a) the population-related, photo-induced magnetooptical Kerr effect and (b) the energy splitting-related magnetooptical Kerr effect around a resonance at $\hbar \omega_{0}$. The inset in (a) shows the calculated temporal profile of the paramagnetic-like Kerr rotation (solid line) and the pulse autocorrelation function (dashed line).

of the ground and excited energy levels, is sometimes called "diamagnetic.", 12 We can expect from the photo-induced Kerr effect in our sample such a diamagneticlike dependence, with a sign reversal of the Kerr rotation at the bandgap energy. On the other hand, optical pumping of a medium with circularly polarized light leads to the selective excitation of one transition, from $m_{j}=-\frac{3}{2}$ to $m_{j}=-\frac{1}{2}$ or from $m_{j}=\frac{3}{2}$ to $m_{j}=\frac{1}{2}$, depending on the helicity of the light polarization. During and after the excitation, the probe pulse itself will be less likely to engage in an already partially pumped transition and, as a result, the optical coefficients of the medium submitted to probe pulses with two circular polarizations of opposite helicities are different. It follows that an additional contribution affects the photo-induced magneto-optical effects. ${ }^{13}$ The simulated spectral and temporal dependences of this contribution for a two-level system are shown on Fig. 2(a). This type of spectral behavior has been named "paramagnetic" and shows a maximum of the Kerr rotation at the interband transition. It should be noted that such a contribution to photo-induced magneto-optical effects due to population changes of excited states was studied in $\mathrm{Cd}_{0.75} \mathrm{Mn}_{0.25} \mathrm{Te}\left(E_{g}=1.95 \mathrm{eV}\right)$ at $\hbar \omega=1.17 \mathrm{eV},{ }^{14}$ and that the relevant effect was quadratic in the intensity of light.

The change of the polarization state of the probe pulse upon reflection from the optically excited sample is described by ${ }^{15}$

$$
\varepsilon_{K}+i \theta_{K} \propto \int d t E^{*}(t)\left[P^{++}(t)-P^{--}(t)\right],
$$

where $\varepsilon_{K}$ is the Kerr ellipticity and $\theta_{K}$ is the Kerr rotation. The nonlinear polarization $P^{++}=P_{x}+i P_{y}$ (respectively, $\left.P^{--}=P_{x}-i P_{y}\right)$ is a linear function of the probe optical electric field and a quadratic function of the optical electric field of the right (respectively, left)-handed polarized pump pulse. Thus the photo-induced effect is expected to be linearly dependent on the pump intensity.

For the experiments a pulsed Ti:sapphire laser was used, with a pulse duration of approximately $100 \mathrm{fs}$ and a repetition rate of $82 \mathrm{MHz}$, in the range of 1.44 to $1.63 \mathrm{eV}$ (Fig. 3). Coherent pump and probe pulses in an intensity ratio of 10:1 were focused on the sample to a spot diameter of about 100 $\mu \mathrm{m}$ for the pump pulse and slightly less for the probe pulse.

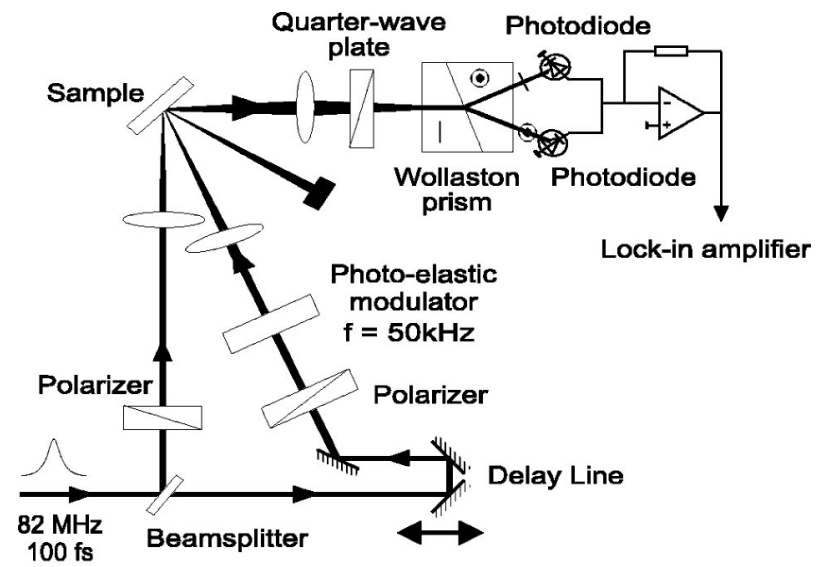

FIG. 3. Experimental setup for time-resolved measurements of the Kerr rotation and ellipticity.

The pump fluence on the sample was of the order of 10 $\mu \mathrm{J} \mathrm{cm}{ }^{-2}$ and at $\hbar \omega=1.52 \mathrm{eV}$ produced a spatially averaged electron density of about $10^{17} \mathrm{~cm}^{-3}$. The angles of incidence of the pump and probe beams with respect to the sample normal were set to $20^{\circ}$ and $40^{\circ}$, respectively. These relatively large angles were chosen in order to minimize coherent contributions such as those observed in four-wave mixing experiments. ${ }^{16}$ The Kerr rotation and ellipticity experienced by the reflected delayed probe pulses are measured as a function of the time delay between pump and probe pulses.

In this kind of experiment, where small transient variations of the polarization are the signal of interest, fast fluctuations of the sample reflectivity due to the excitation and relaxation of carriers are a common burden. Their influence was minimized by using a very sensitive balanced detection scheme. The circular polarization of the pump pulses was modulated at a frequency of $50 \mathrm{kHz}$ between left-handed and right-handed helicity with a photo-elastic modulator (PEM), without changing their intensity. A Wollaston prism was then used to split the reflected probe beam into two spatially separated components with polarization directions orthogonal to each other. The corresponding intensities were detected with a pair of equivalent photodiodes and then subtracted through a differential amplification circuit. The output signal, proportional to the Kerr rotation $\theta_{K}$ (or to the Kerr ellipticity $\varepsilon_{K}$ measured with a Babinet-Soleil compensator as a quarter-wave plate in front of the prism), was then measured at $50 \mathrm{kHz}$ with a lock-in amplifier. This sensitive balanced detection technique allowed for the measurement of very tiny pump-induced polarization changes in the sample down to $0.2 \mu \mathrm{rad}$. The spectral profile of the laser radiation was also monitored with an optical multichannel analyzer, and a photon energy range of about $0.02 \mathrm{eV}$ was measured.

Experimentally, a linear dependence of the photo-induced Kerr effect on the pump intensity was found for fluences ranging from 1 to $10 \mu \mathrm{J} \mathrm{cm}{ }^{-2}$. The maximum of the effect was observed for a circular pump polarization in agreement with the theoretical background discussed above. Typical relaxation dynamics of the Kerr rotation in CdTe are shown in Fig. 4 for optical excitation below (a) and above (b) the 

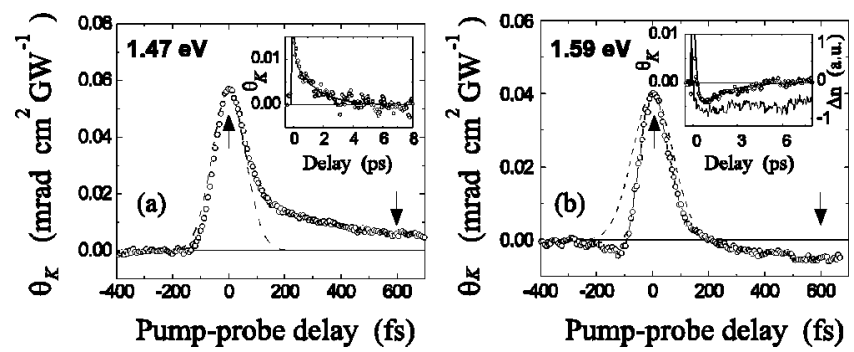

FIG. 4. Relaxation dynamics of the Kerr rotation $\theta_{K}$ in CdTe (a) below and (b) above the band-gap energy. The autocorrelation function of the pulse is indicated with dashed lines. The insets show the long-term dependence of the rotation (circles). For comparison, the inset in (a) shows the decay of the photo-induced linear birefringence due to carrier dynamics (dotted line).

room-temperature band-gap energy $E_{g}=1.52 \mathrm{eV}$. In both cases, the short-term behavior of $\theta_{K}$ is presented, as well as the long-term decay of the signal up to complete relaxation, in this case after 5 to 6 ps (see insets). A similar relaxation behavior was observed for the Kerr ellipticity $\varepsilon_{K}$. The temporal shape of the autocorrelation function of the pump pulses, as deduced from the measured spectral profiles, has been included for comparison (dashed lines). Clearly, the initial peak of $\theta_{K}$ does not exactly follow the shape of this autocorrelation function, but shows a fast relaxation component up to a pump-probe time delay $\Delta t$ of approximately 600 fs, after which a slower relaxing contribution totally decays within a few picoseconds. For energies above the band-gap, a competition between the slower and the ultrafast relaxation contributions was observed during the overlap of the pulses. Around zero delay, i.e., when pump and probe pulses coincide in time at the sample surface - the fast relaxing part dominates the response.

Within the photon energy range of 1.44 to $1.63 \mathrm{eV}$ the experimentally deduced decay times were found between 100 and 200 fs for the faster relaxation part, and between 1 and $2.5 \mathrm{ps}$ for the slower component. A possible artifact in such polarimetric measurements is that one not only modulates the polarization of the pump pulses, but also their intensity, which would induce in the sample a transient linear birefringence not related to the spin dynamics. We investigated this possibility by placing an additional polarizer behind the PEM. As a result the intensity of the pump pulses was intentionally modulated, while their polarization was kept linear at an angle of $30^{\circ}$ relatively to the probe polarization. The resulting dynamics of the photo-induced birefringence $\Delta n$, as shown in the inset of Fig. 4(b) (dotted line) proved to be much slower than both components of the Kerr dynamics, confirming that the latter do have a magnetooptical origin.

The amplitudes of $\theta_{K}$ and $\varepsilon_{K}$ are presented as a function of the photon energy in Fig. 5, both for the short term 5(a) and the longer term 5(b). More specifically, as indicated by the solid arrows in Fig. 4, the short-term value was measured for each photon energy at the signal maximum, when both relaxing components are present, i.e., at $\Delta t=0$, whereas the longer-term value was taken at $\Delta t=600 \mathrm{fs}$, after the fast relaxation component has totally vanished. The spectral dependence for the Kerr rotation $\theta_{K}$ in the short term clearly shows a maximum near the interband transition at $1.52 \mathrm{eV}$.
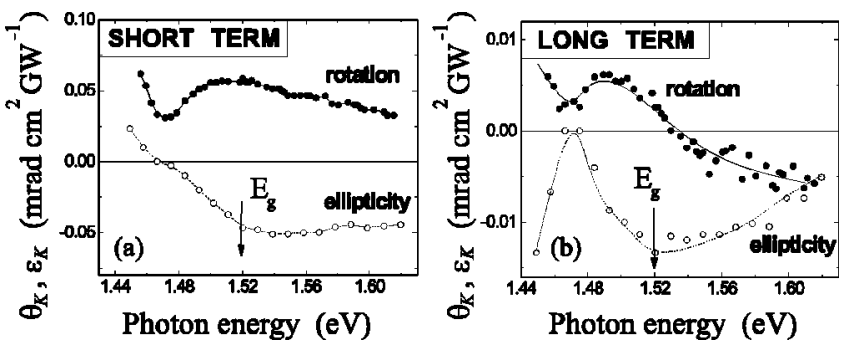

FIG. 5. Spectral dependence of the Kerr rotation $\theta_{K}$ and ellipticity $\varepsilon_{K}$ in CdTe (a) in the short term and (b) in the long term for pump-probe delays corresponding to the solid arrows in Fig. 4. Lines are guides to the eye.

Moreover, the signal tends to increase towards a second maximum when the photon energy decreases below $1.44 \mathrm{eV}$. This is likely due to a transition at an energy $E_{i}$ between the top of the valence band and a bound impurity level within the band gap. ${ }^{17}$ The spectra for $\theta_{K}$ and $\varepsilon_{K}$ are expected to obey the Kramers-Kronig relations, i.e., $\varepsilon_{K}$ ought to experience a sign reversal where $\theta_{K}$ reaches an extremum. However, as can be seen from Fig. 5(a), the short-term spectral dependence for the ellipticity actually changes sign below the interband transition at $1.52 \mathrm{eV}$. This may indicate that the transition at $E_{i}$ dominates over the interband and excitonic transitions. With this in mind, the short-time spectral dependencies of $\theta_{K}$ and $\varepsilon_{K}$ do obey the Kramers-Kronig relations and follow a paramagnetic-like behavior. The domination of the transition at $E_{i}$ arises from a peculiarity of the photoinduced magneto-optical Kerr effect. It can be shown that $\theta_{K}$ and $\varepsilon_{K}$ not only depend on the probability of a given transition, but also greatly decrease when the decay time from the excited state increases. We indeed observed a strong decrease of the decay time of the rapid contribution between 1.44 and $1.48 \mathrm{eV}$. Thus the transition at $E_{i}$ bears a larger spectral weight than interband and excitonic transitions in this spectral region, although its probability is smaller.

The Kerr rotation in the longer term changes sign at the interband transition energy while the ellipticity shows an extremum at this point. Thus ellipticity and rotation once again correspond to one another with respect to the KramersKronig relations, but they now show a diamagneticlike spectral behavior. Thus, an evolution of the magneto-optical Kerr effect spectral dependence from a paramagnetic to a diamagnetic type has taken place during the fast relaxation process.

These spectra exhibit a distinct resonance behavior, which is typical for a two-level system. Thus, we assume that excitonic transitions play a considerable role in the observed photo-induced Kerr effect. The deviation from a resonance behavior, which appears in the asymmetry of the spectral dependences around the resonance, can be due to the interband transitions. These transitions are usually described by invoking an inhomogenously broadened two-level system. ${ }^{11}$

According to the qualitative mechanisms described above, for pump-probe delays up to $600 \mathrm{fs}$ the paramagneticlike behavior of the photo-induced Kerr effect in CdTe can be attributed to the difference in populations of excited states with a magnetic quantum number $m_{j}$ equal to $-\frac{1}{2}$ and $+\frac{1}{2}$, respectively. After this paramagneticlike contribution has completely decayed the diamagneticlike spectral dependence starts to dominate the relaxation in the picosecond range. 
This diamagneticlike contribution is caused by the repulsive interaction among the carriers due to the Pauli exclusion principle $^{18}$ and the decay of this contribution is related to electron-spin relaxation. The observed decay times are in reasonable agreement with data reported elsewhere. ${ }^{6}$

In contrast, several relaxation processes can be responsible for the ultrafast paramagnetic-like contribution to the Kerr effect dynamics. Around the band-gap energy exciton ionization is a possible relaxation process, but this contribution is essentially attributed to phase-space filling effects ${ }^{19}$ and its relaxation is related to the energy relaxation of the photo-excited electrons towards the bottom of the conduction band, through the emission of LO phonons. For a relatively high density of electron-hole pairs photo-excited by the pump pulse, carrier-carrier scattering is also a significant energy relaxation process. ${ }^{20}$ But the linear dependence of the Kerr effect on the intensity proves that carrier-carrier interactions do not contribute significantly to the relaxation.

In conclusion, the dynamics of the photo-induced magneto-optical Kerr effect in CdTe at room temperature was studied in the spectral range of 1.44 to $1.63 \mathrm{eV}$ with a subpicosecond resolution using a sensitive polarimetric technique. Two relaxation processes with respective decay times of 100 to 200 fs and 1 to 2.5 ps were observed. It was found that during the fast relaxation process, the spectral dependence of the effect changes from a paramagnetic to a diamagnetic type. Several ultrafast processes, such as the redistribution of electrons within the conduction band or exciton ionization near the band-gap can be responsible for the faster decay, whereas the longer one can be attributed to electronspin relaxation.

The authors are grateful to B. Koopmans and A. V. Petukhov for fruitful discussions. This work was supported in part by NWO, INTAS, RFBR, and the EU TMR Network NOMOKE. A.V.K. thanks the Robert Havemann Foundation for financial support.
${ }^{1}$ J. M. Kikkawa and D. D. Awschalom, Nature (London) 397, 139 (1999); I. Malajovich, J. M. Kikkawa, D. D. Awschalom, J. J. Berry, and N. Samarth, Phys. Rev. Lett. 84, 1015 (2000).

${ }^{2}$ S. Bar-Ad and I. Bar-Joseph, Phys. Rev. Lett. 68, 349 (1992).

${ }^{3}$ C. Buss, R. Pankoke, P. Leisching, J. Cibert, R. Frey, and C. Flytzanis, Phys. Rev. Lett. 78, 4123 (1997).

${ }^{4}$ J. Güdde, U. Conrad, V. Jähnke, J. Hohlfeld, and E. Matthias, Phys. Rev. B 59, R6608 (1999).

${ }^{5}$ T. Elsaesser, J. Shah, L. Rota, and P. Lugli, Phys. Rev. Lett. 66, 1757 (1991).

${ }^{6}$ B. Koopmans and W. J. M. de Jonge, Appl. Phys. B: Lasers Opt. 68, 525 (1999).

${ }^{7}$ L. D. Landau and E. M. Lifshitz, Electrodynamics of Continuous Media (Pergamon Press, Oxford, 1984).

${ }^{8}$ D. T. Pierce and F. Meier, Phys. Rev. B 13, 5484 (1976).

${ }^{9}$ M. I. Dyakonov and V. I. Perel, in Optical Orientation, edited by F. Meyer and B. P. Zakharchenya (North-Holland, Amsterdam, 1984).

${ }^{10}$ W. H. Knox, R. L. Fork, M. C. Downer, D. A. B. Miller, D. S. Chemla, C. V. Shank, A. C. Gossard, and W. Wiegmann, Phys. Rev. Lett. 54, 1306 (1985).
${ }^{11}$ J. Shah, Ultrafast Spectroscopy of Semiconductors and Semiconductor Nanostructures (Springer, Berlin, 1996).

${ }^{12}$ A. K. Zvezdin and V. A. Kotov, Modern Magnetooptics and Magnetooptical Materials (IOP, Bristol, 1997).

${ }^{13}$ A. G. Aronov and E. L. Ivchenko, Fiz. Tverd. Tela 15, 231 (1973) [Sov. Phys. Solid State 15, 160 (1973)].

${ }^{14}$ J. Frey, R. Frey, and C. Flytzanis, Phys. Rev. B 45, 4056 (1992).

${ }^{15}$ Th. Östreich, K. Schönhammer, and L. J. Sham, Phys. Rev. Lett. 75, 2554 (1995); L. J. Sham, J. Magn. Magn. Mater. 200, 219 (1999).

${ }^{16}$ A. Leitenstorfer, A. Lohner, K. Rick, P. Leisching, T. Elsaesser, T. Kuhn, F. Rossi, W. Stolz, and K. Ploog, Phys. Rev. B 49, 16372 (1994).

${ }^{17}$ B. Montegu, A. Laugier, and R. Triboulet, J. Appl. Phys. 56, 3061 (1984).

${ }^{18}$ L. Viña, L. Muñoz, E. Pérez, J. Fernández-Rossier, C. Tejedor, and K. Ploog, Phys. Rev. B 54, R8317 (1996).

${ }^{19}$ J. A. Kenrow, K. El Sayed, and C. J. Stanton, Phys. Rev. B 58, R13 399 (1998).

${ }^{20}$ M. Betz, G. Göger, A. Leitenstorfer, K. Ortner, C. R. Becker, G. Böhm, and A. Laubereau, Phys. Rev. B 60, R11 265 (1999). 